\title{
Combined Partial Knee Arthroplasty
}

\author{
Amy Garner and Justin Cobb
}

\section{Abbreviations}

ACL Anterior cruciate ligament

BCA-L Bicompartmental knee arthroplasty (lateral)

BCA-M Bicompartmental knee arthroplasty (medial)

Bi-UKA Bi-unicondylar knee arthroplasty

CPKA Combined partial knee arthroplasty

EQ-5D EuroQol-5D Index of Quality of Life

OKS Oxford knee score

PFA Patellofemoral arthroplasty

PFJ Patellofemoral joint

PKA Partial knee arthroplasty

TCA Tricompartmental knee arthroplasty

TKA Total knee arthroplasty

UKA Unicompartmental knee arthroplasty

\section{Key Points}

- Bone- and cruciate-preserving alternative to total knee arthroplasty.

- High-functioning arthroplasty option when the anterior cruciate ligament is intact.

- Unlinked components offer patient-specific surgery with conventional implants.

- Suitable for young, active, high-demand patients in the primary setting.

- Addition of components to existing partial knee arthroplasty offers a safer, less invasive alternative to the revision to total knee arthroplasty.

\subsection{Introduction}

Arthrosis commonly affects a single compartment of the knee, but may present with two or even three compartments affected. Wear to the medial tibiofemoral compartment is ten times more common than that in the lateral tibiofemoral compartment; primary patellofemoral joint (PFJ) arthrosis is least common [1,2]. Bicompartmental disease is present in $59 \%$ of those with gonarthrosis [3]. In one study, $40 \%$ of patients over 50 years old with knee pain had radiographic evidence of combined medial compartment and PFJ wear, 24\% had isolated PFJ arthrosis, whilst only $4 \%$ had isolated tibiofemoral arthrosis [4]. Degeneration of all three compartments simultaneously is rare [2]. Consequently, removal of
A. Garner $(\bowtie)$

MSk Lab, Imperial College London, London, UK

Health Education, Kent, Surrey and Sussex, London, UK

Royal College of Surgeons of England and Dunhill Medical Trust, London, UK

e-mail: a.garner@imperial.ac.uk

J. Cobb

MSk Lab, Imperial College London, London, UK e-mail: j.cobb@imperial.ac.uk 
healthy tissue in total knee arthroplasty (TKA) is common. The anterior cruciate ligament (ACL) is present in $78 \%$ of cases of patients undergoing primary knee replacement [5]. The fundamental role of the ACL in knee stability and functional gait is well described [6]; however, regardless of its functional integrity, it is resected in almost all TKAs.

TKA is associated with up to $20 \%$ patient dissatisfaction [7], significant peri-operative risk [8] and limited function when the ACL is sacrificed. However, in the absence of an effective alternative, TKA remains the standard treatment for multicompartment arthrosis [9]. Combined partial knee arthroplasty (CPKA) is the collective term for multiple partial knee arthroplasties (PKAs) used together within the same knee, preserving healthy compartments and functional cruciate ligaments as an alternative to TKA [10]. Four combinations of CPKA exist (Fig. 21.1): Bicompartmental knee arthroplasty (BCA) refers to a patellofemoral arthroplasty (PFA) in combination with either a medial (BCA-M) or lateral (BCA-L) unicompartmental knee arthroplasty (UKA), whilst bi-unicondylar knee arthroplasty (Bi-UKA) describes an ipsilateral medial and lateral UKA [10]. All three used in combination are referred to as a tricompartmental knee arthroplasty (TCA). CPKA is not a new idea. The original Gunston knee, Charnley's 'load angle inlay' knee, the Marmor modular knee, the Cartier knee and the Oxford unicompartmental knee systems all followed a biunicondylar configuration.

In the presence of a functional ACL, multicompartment arthrosis can be addressed through single-stage CPKA. Alternatively, a patient previ- ously treated with a single PKA may be converted to a CPKA in a further operation, in the event of subsequent native compartment degeneration. The advantage of the latter, 'staged' procedure, is that the second operation may be considered a primary PKA with the benefits of a shorter hospital stay and reduced perioperative risk [8]. Advocates of CPKA argue that, in tailoring the surgery to the exact disease pattern of the patient, a second procedure may never become necessary and healthy bone and soft tissues are preserved whilst minimising the risk to the patient and optimising function and satisfaction. If a second surgery in PKA involves conversion to a standard primary TKA, this is a relatively straightforward process, especially if a kinematic technique is employed [11], which may delay or prevent the need for revision to the TKA. Opponents, however, argue that if the entire knee is replaced in the first instance, the patient may avoid the need for a second procedure altogether. Using two implants in combination, together with the potential need for additional hospital admissions, has a financial implication, though this additional cost may be offset by shorter hospital stays following both the primary and revision procedure, and fewer perioperative complications.

\subsection{Case 1}

A 64-year-old male presented with antero-medial right knee pain and difficulty standing up from a chair and walking up the stairs. He reported night pain, occasional giving way and now walks with a stick, but is keen to return to playing tennis. On examination, he had a moderate effusion and
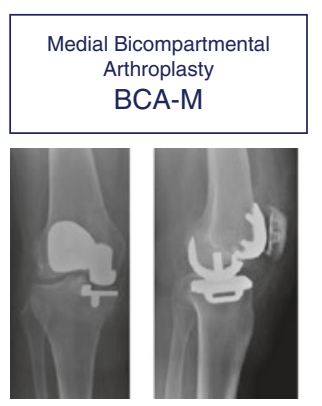
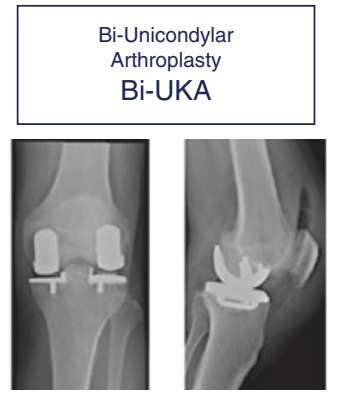
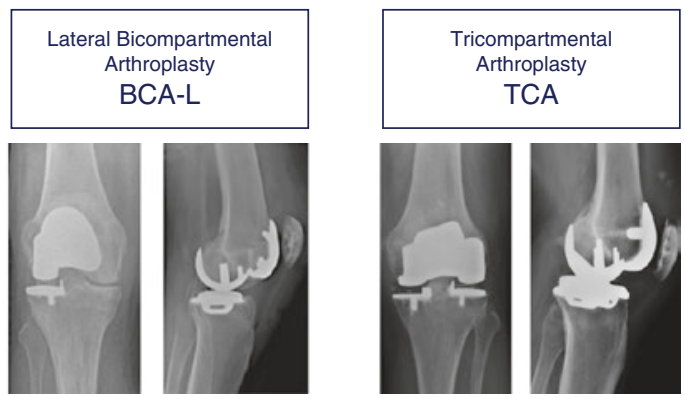

Fig. 21.1 Classification of combined partial knee arthroplasty (CPKA) 
correctable varus deformity. Range of motion was 5-130 ${ }^{\circ}$. Lachman and Anterior Drawer tests were negative. He had extrusion of the medial meniscus, but the lateral meniscus did not extrude on valgus stress. Pre-operative radiographs (Fig. 21.2) show varus alignment with significant loss of joint space in the medial compartment, osteophytes and subchondral sclerosis. There is some medial translation of the tibia on the femur. There is significant arthrosis of the lateral facet of the patellofemoral joint. The lateral compartment is well preserved with no evidence of arthrosis. On the lateral view, the ACL appears to be functional with no evidence of anterior translation of the tibia on the femur.
The patient was presented with the options for surgical management (Table 21.1) but prioritised high levels of function and opted for BCAM. The patient was positioned supine on the operating table with a side support and foot support to hold the knee at $90^{\circ}$ of flexion. A midline incision and medial parapatellar approach were used to access the joint. The lateral compartment was inspected and found to be disease-free. The ACL was intact. The UKA-M was undertaken first to correct the alignment and left with trial implants whilst the trochlea was prepared. The patella button was trialled to ensure it tracked smoothly over the trochlear component and
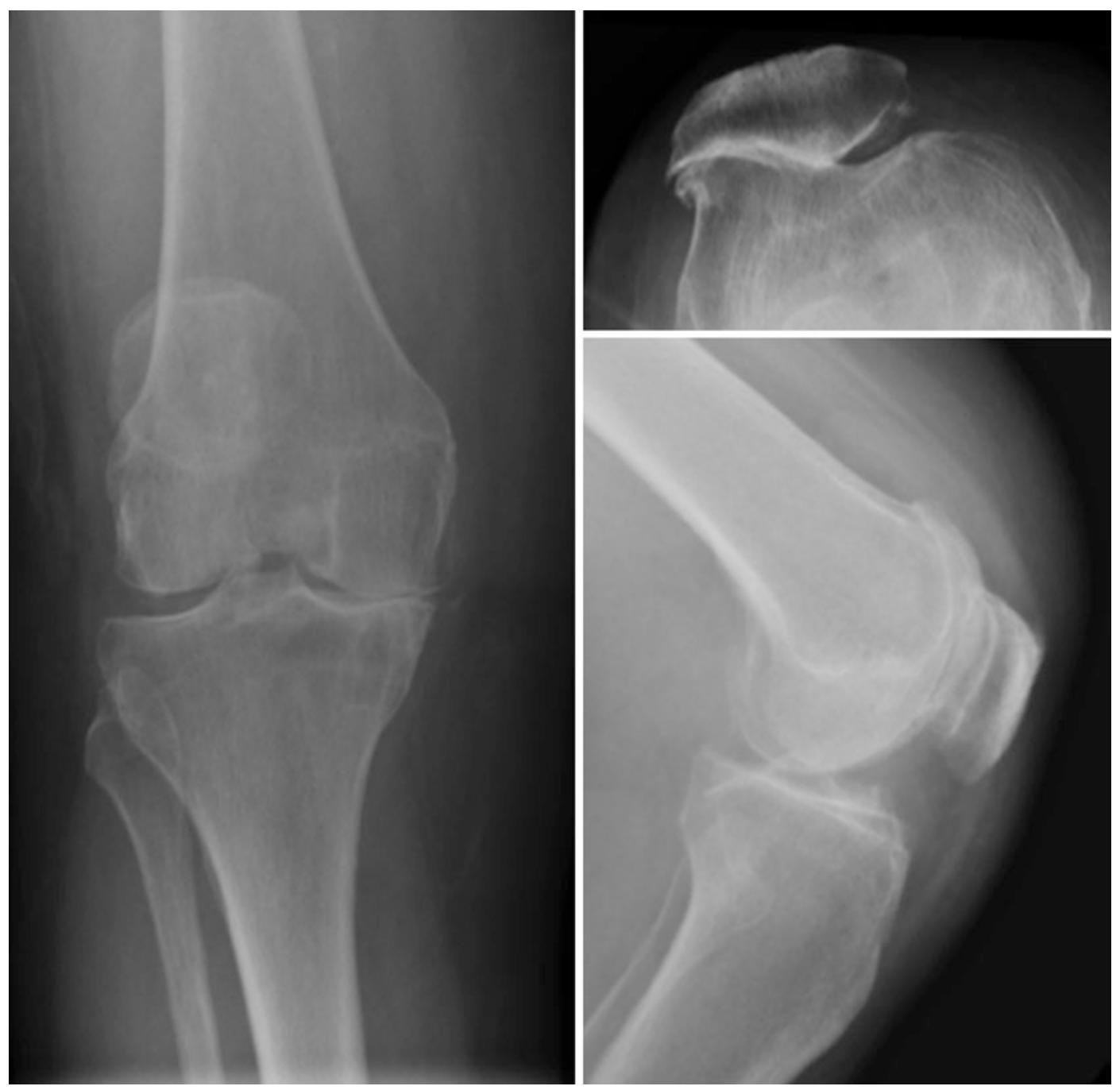

Fig. 21.2 Pre-operative radiographs, Case 1 
Table 21.1 Options for surgical management of medial compartment with lateral facet patellofemoral arthrosis

\begin{tabular}{l|l|l}
\hline $\begin{array}{l}\text { Management } \\
\text { option }\end{array}$ & Advantages & Disadvantages \\
\hline TKA & $\begin{array}{l}\text { Technically straightforward } \\
\text { Widely available } \\
\text { Lower risk for revision } \\
\text { No risk of native compartment } \\
\text { degeneration }\end{array}$ & $\begin{array}{l}\text { ACL sacrifice-compromised function } \\
\text { Up to 20\% dissatisfaction } \\
\text { Higher perioperative risks } \\
\text { Longer hospital stay } \\
\text { Removal of healthy bone (lateral compartment) }\end{array}$ \\
\hline UKA-M & $\begin{array}{l}\text { Bone preserving } \\
\text { Short hospital stay } \\
\text { Lower perioperative risks } \\
\text { Least traumatic } \\
\text { ACL preserving-higher function }\end{array}$ & $\begin{array}{l}\text { Does not address patellofemoral arthrosis } \\
\text { Higher revision risk } \\
\text { Risk of further degeneration necessitating revision }\end{array}$ \\
\hline PFA & $\begin{array}{l}\text { Bone preserving } \\
\text { Short hospital stay }\end{array}$ & $\begin{array}{l}\text { Does not address medial tibiofemoral arthrosis } \\
\text { Will not correct alignment } \\
\text { Risk of further degeneration necessitating revision } \\
\text { Highest revision risk } \\
\text { (not recommended in isolation for bi-compartmental } \\
\text { arthrosis) }\end{array}$ \\
\hline BCA-M & $\begin{array}{l}\text { Treats all affected compartments } \\
\text { Bone preserving } \\
\text { Will correct alignment } \\
\text { ACL preserving-highest function }\end{array}$ & $\begin{array}{l}\text { Risk of revision if lateral compartment fails } \\
\text { Unknown revision rates (likely higher than TKA) } \\
\text { Unknown perioperative risk (likely lower than TKA) } \\
\text { Technically challenging-few surgeons perform it } \\
\text { Higher implant costs }\end{array}$ \\
\hline
\end{tabular}

did not catch on the femoral component of the medial UKA. A final check to ensure the trochlear is well-seated, flush with the neighbouring cartilage, is made, to ensure the patella button transitions smoothly between implants. Care is taken not to damage the cartillage betwen the implants during bone preparation. Whilst balancing an UKA in the supine position is more difficult than in a 'dangling' support, it improves the technical ease of the PFA, so it is preferred for simultaneous BCA-M. All components were implanted simultaneously after all of the bony cuts had been performed. Tourniquet time was $64 \mathrm{~min}$ (surgeon average for UKA is $45 \mathrm{~min}$ ). The patient recovered without peri-operative complication and was discharged within $48 \mathrm{~h}$ of surgery. Within 4 months of surgery, he had returned to full function including playing tennis twice per week. His Oxford Knee Score was 44 at 6 months, rising to 47 at 12 months and continuing at 476 years post-surgery. Post-operative radiographs (Fig. 21.3) show a mobile-bearing UKA-M and onlay PFA in situ, with correction of the varus deformity and tibial translation. The lateral compartment is preserved, and the ACL appears functional, and the patella button tracks adequately over the resurfaced trochlea.

\subsection{Function Post-CPKA}

A number of studies and expert opinions emphasise the benefits of BCA [12], including superior performance in strenuous activities such as stair climbing and jogging, compared to TKA, in part due to restored isokinetic quadriceps function [13]. High function, independent rising from a chair and reciprocal stair ascent is seen rapidly and consistently after BCA $[14,15]$. Kinematics and gait patterns associated with BCA are similar to those of healthy controls $[14,16]$. Compared to TKA, several studies report that patients with BCA have higher levels of satisfaction and comfort following surgery $[17,18]$, with good or excellent pain outcomes reported up to 12 years post-operatively in $85 \%$ of patients, $92 \%$ of whom reported satisfactory pain relief [19]. Patients experience less intra-operative blood loss [20] and greater postoperative range of movement [21] compared to matched groups undergoing TKA.

In Case 1, the femur has been addressed through two unlinked components. A significant advantage of unlinked CPKA is that each component can be orientated according to the specific anatomy of the compartment, effectively allowing the surgeon to create a custom fit, using 'off-the- 

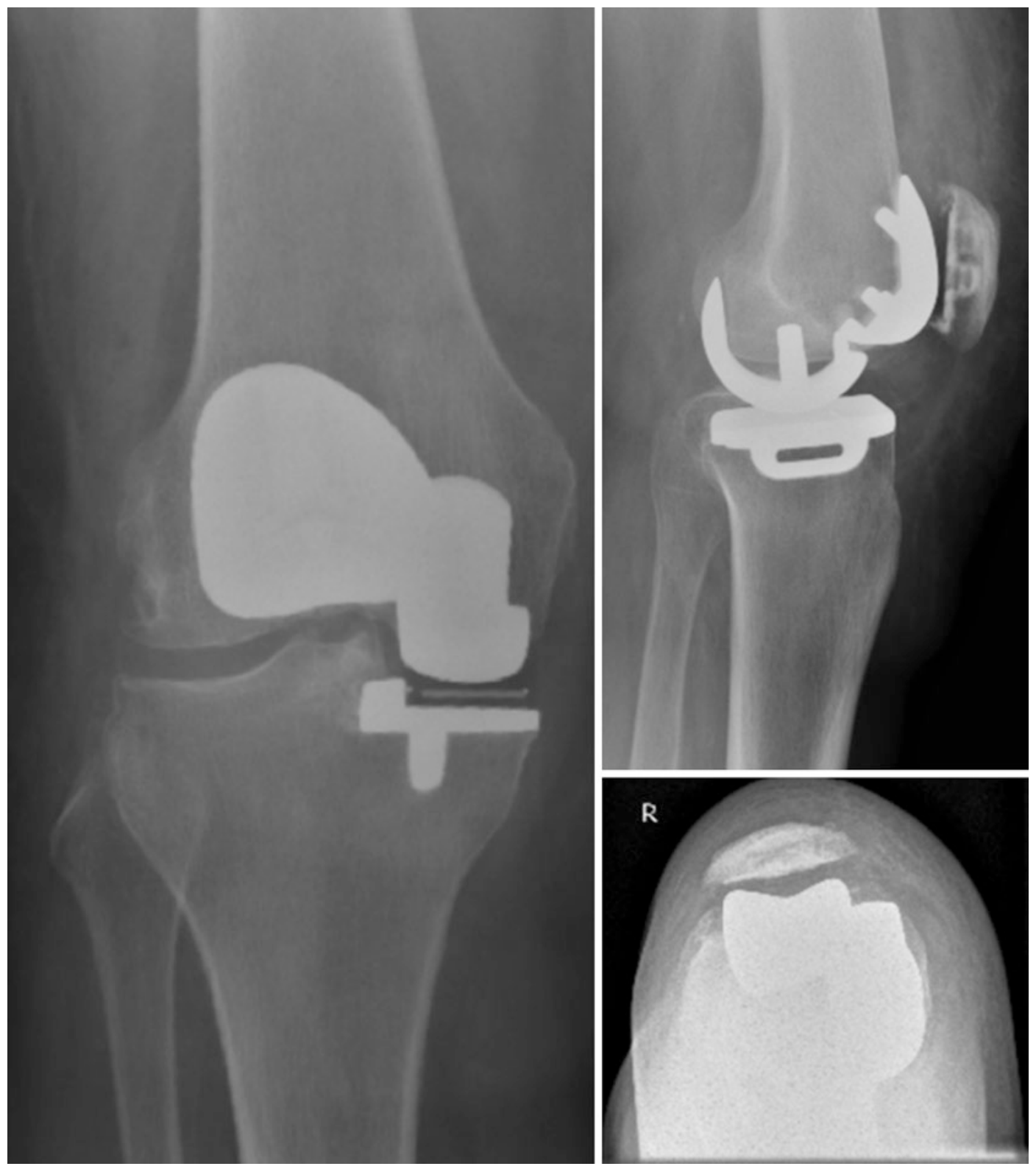

Fig. 21.3 Post-operative radiographs, Case 1, with BCA-M in situ

shelf' implants [22]. An alternative is to use a monolithic femoral component, which simultaneously resurfaces both the condyle and the trochlea. Whilst monolithic femoral components are theoretically easier to implant, early examples including the Journey Deuce (Smith and Nephew Inc., Memphis, TN, US) performed very poorly, blighted by high rates of early revision (Fig. 21.4). Malalignment, sizing difficulties, poor durability, anterior knee pain, limited range of movement and tibial component fractures were all cited as causes for early failure [13]. In one short-term study, a $12 \%$ revision rate was reported, with $25 \%$ of patients complaining of anterior knee pain [23]. In another study of 25 Journey Deuce, three were revised - two for fractured tibial trays and one for patella instability [24]. These reports, plus evidence of tibial subsidence, contributed to the US Food and Drug Administration's decision to recall the Journey Deuce prosthesis in 2010. 

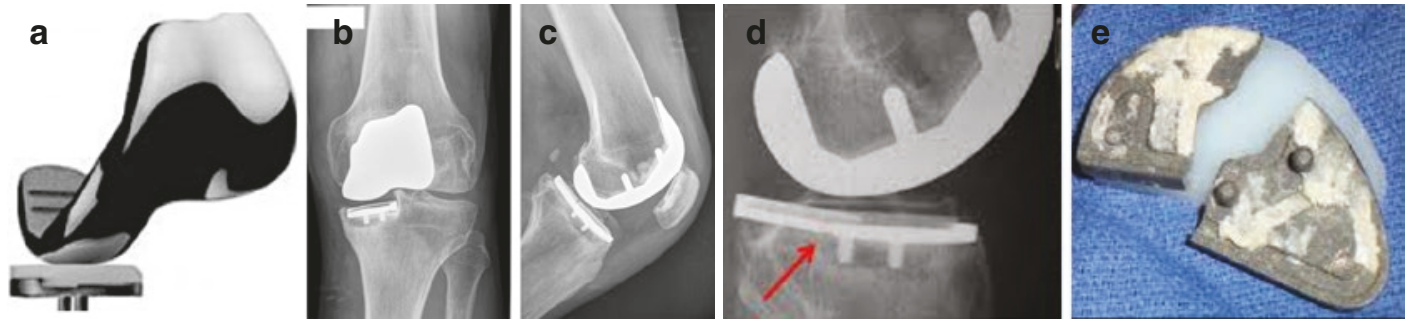

Fig. 21.4 Monolithic Journey Deuce (Smith and Nephew Inc., Memphis, TN, US) (a) with tibial component subsidence $(\mathbf{b}, \mathbf{c})$ and tibial baseplate fracture $(\mathbf{d}, \mathbf{e})[24,25]$

Contemporary monolithic designs are utilising assistive technologies including 3D-printed patient-specific instrumentation, robotics and navigation to help improve alignment accuracy and decrease the technical demands of this procedure [26] which may lead to a resurgence in interest in linked components. Modular CPKA may allow the surgeon more freedom to make subtle adjustments according to the distal femoral geometry of the femur, with promising results but a steep learning curve [21, 23, 27-29]. Some early modular BCA-M had a $46 \%$ incidence of disease progression or radiographic evidence of loosening by 17 years post-operation, likely due to poor-quality polyethylene and crude instrumentation necessitating a "free-hand" technique [30]. Aseptic loosening of the PFA implant was the main cause of failure in 20/27 revised BCA-M [30]. Experience with BCA failure, however, provided much evidence that conversion to TKA was typically straightforward, using primary TKA implants [29, 31-33]. Secondgeneration anterior-cut (onlay design) cemented patellofemoral components are associated with improved clinical and biochemical outcomes [34-36]. Unlinked components enable more accurate alignment [34].

\subsection{Case 2}

A 54-year-old male presented with lateral joint knee pain and difficulty walking on slopes. He has been a keen hill walker for many years. He reports swelling in the knee and now requires daily anti-inflammatory medications to walk short distances. On examination, he has a good range of movement but extrusion of the lateral meniscus. Lachman test was negative, and the knee felt stable, with no medial meniscal extrusion on varus stressing.

Weight-bearing radiographs (Fig. 21.5) demonstrate a valgus right knee, with Ahlback grade IV loss in the lateral compartment with some medial opening. There is severe degeneration of the lateral facet of the PFJ. The ACL appears functional on the lateral radiograph, with no evidence of anterior translation of the tibia on the femur.

This young patient prioritised high function and opted for single-stage BCA-L. A midline incision was made, followed by a lateral parapatellar arthrotomy. Additional care was taken to sublux the patella medially to enable adequate exposure. Extending the arthrotomy into the quadriceps tendon is sometimes necessary to improve the view, but may increase the associated morbidity of the procedure. The medial compartment was found to be well preserved, and the ACL was functional and intact. On the lateral side, it is particularly important to ensure the patella has a smooth transition between the femoral components of the UKA and PFA and the femoral condylar cartilage for accurate tracking. Care should be taken not to over-resect bone from the distal femur, if required, to avoid impingement of the UKA bearing in full extension. The patient experienced no peri-operative complications and returned to hill walking within 6 months. His Oxford Knee Score was 44 at 12 months post-surgery, EQ-5D 0.95/1. Post-operative radiographs (Fig. 21.6) demonstrate the BCA-L in situ and confirm that the medial compartment is preserved and alignment corrected. The patella tracks centrally across the resurfaced trochlea. In this case a mobile-bearing lateral UKA was used to prioritise high function, 
Fig. 21.5 Pre-operative radiographs, Case 2
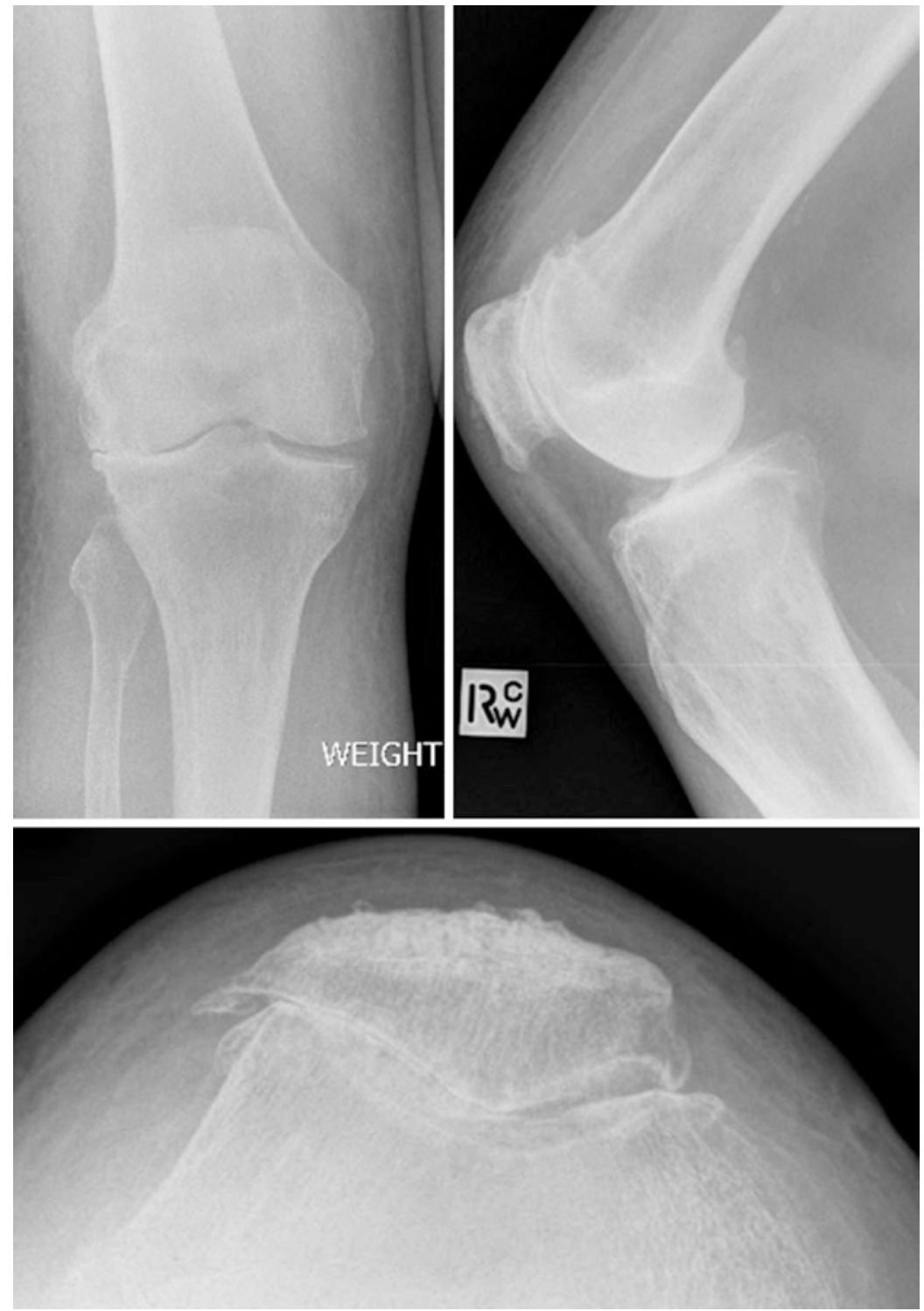

but if concern for the risk of bearing dislocation is present, a fixed bearing device may be more appropriate.

\subsection{Case 3}

An 82-year-old lady presents with knee pain 14 years following a medial UKA. She now requires a walking stick but can stand from a chair and use the stairs without particular difficulty. She has diabetes mellitus type II controlled with insulin, cardiac stents and hypertension and had a transient ischaemic attack 5 years ago. On examination, she has a moderate effusion, correctable valgus deformity of $<10^{\circ}, 0-120$ range of movement and some anterior-posterior laxity; but the medial UKA appears stable and functional. Pre-operative radiographs (Fig. 21.7) demonstrate a well-fixed medial UKA but failure of the lateral compartment. The patellofemoral compartment is relatively well preserved, and the ACL appears functional.

Progression of lateral compartment $\mathrm{OA}$ in patients with medial arthrosis is very rare in the absence of surgical intervention [37, 38]. 
Fig. 21.6 Postoperative radiographs, Case 2, demonstrating a BCA-L in situ
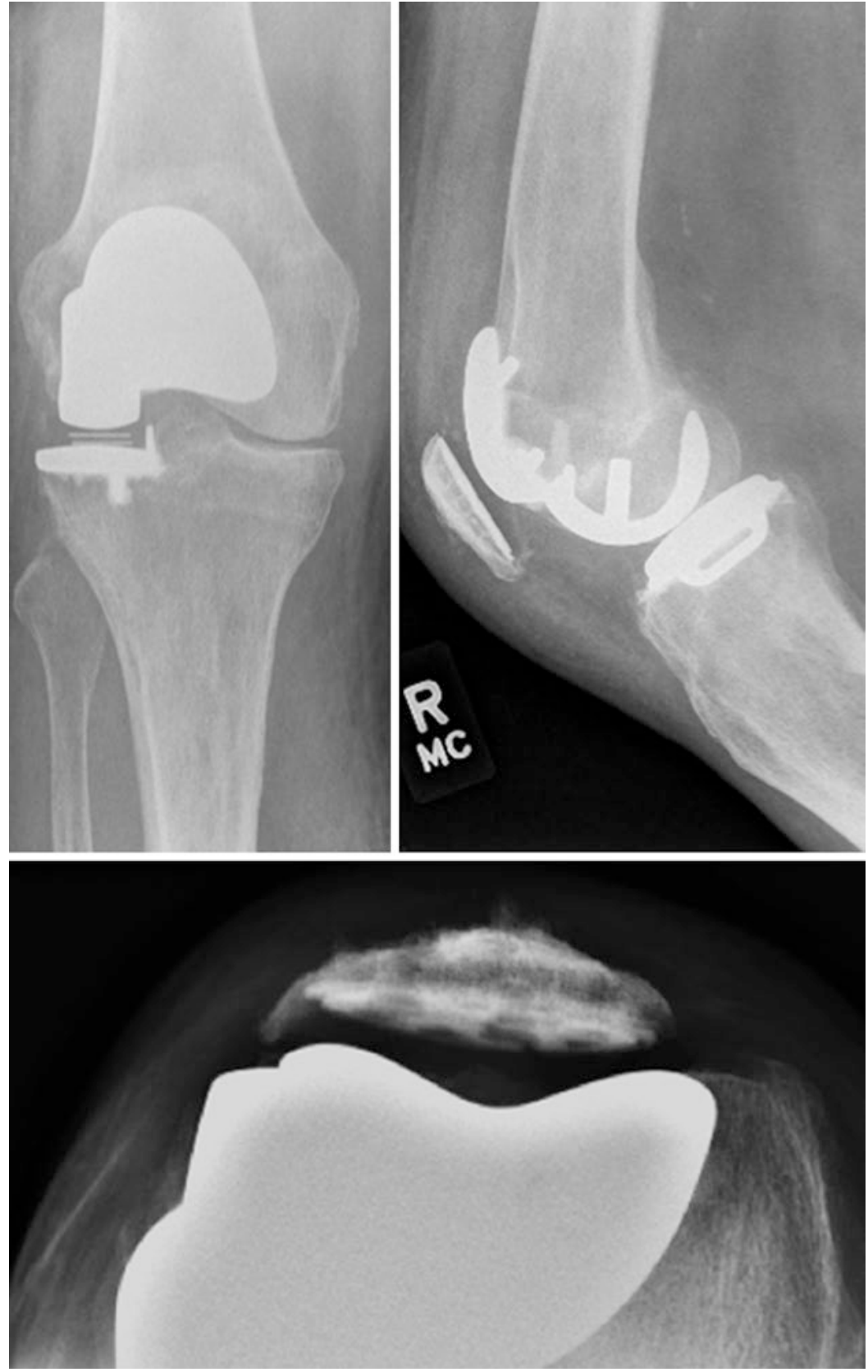

After a medial UKA, lateral arthrosis is often cited as a reason for failure and revision to TKA [22]. However, multiple studies from the Oxford Group and the National Joint Registry, using data from 15- to 20-year follow-up studies, place the revision rate as between 2.3 and $2.6 \%$ [39-41], whilst our own group reported 64 knees with no polyethylene bearing dislocations [42].
The surgical options for managing Case 3's newly degenerate compartment are to remove the well-fixed, high-functioning medial UKA, sacrifice the remaining function of the ACL and patellofemoral compartment and convert to a TKA or leave the medial UKA untouched and 'convert' to a Bi-UKA through the addition of a lateral UKA [43]. Revision to TKA is commonly performed across the world, but carries 

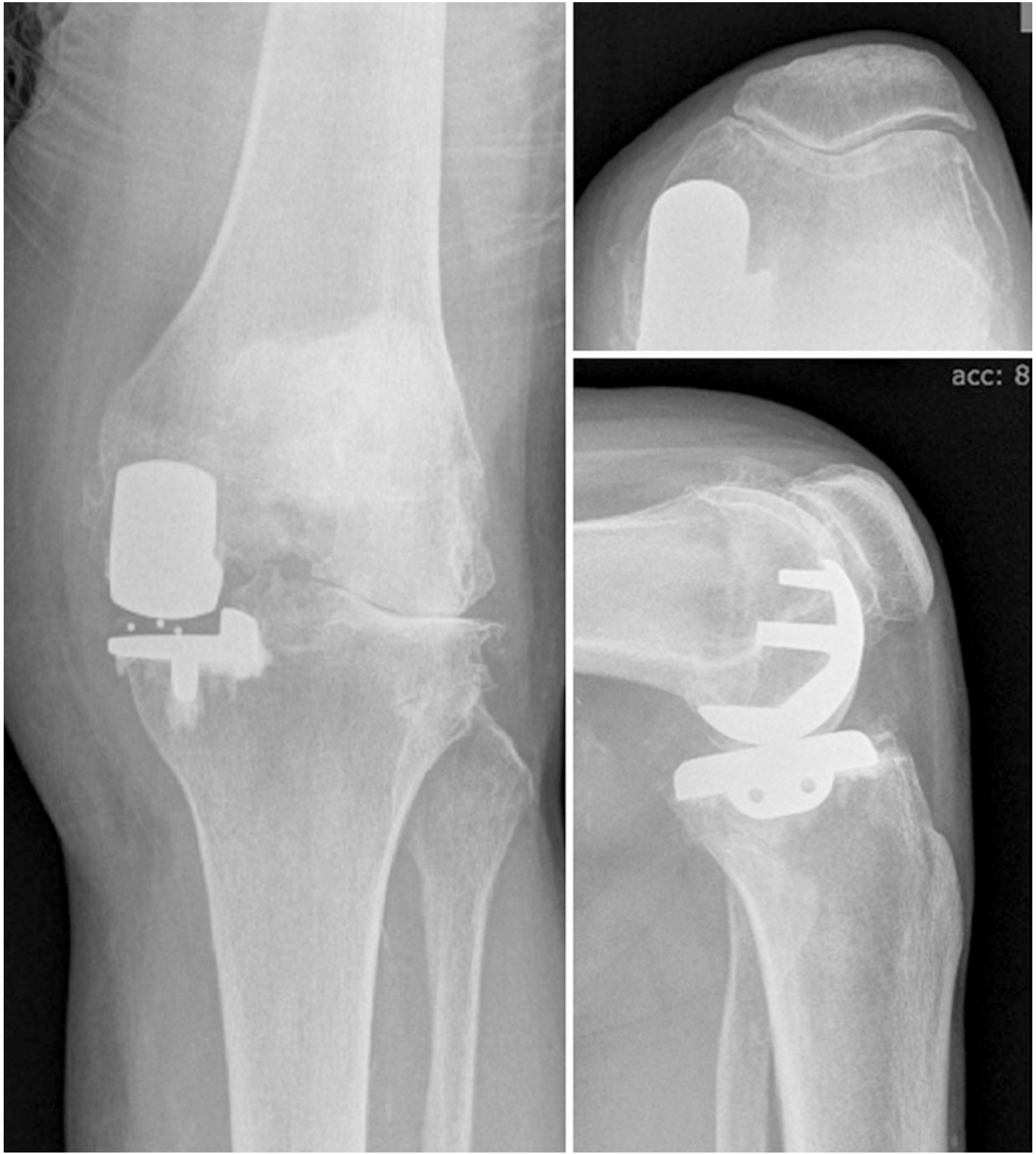

Fig. 21.7 Pre-operative radiographs, Case 3, demonstrating a UKA-M in situ

significant peri-operative risk, requiring a large surgical exposure, the risk of bone loss during implant removal and significant peri-operative risk of stroke, myocardial infarction or death [8]. Although conversion to Bi-UKA would be regarded by joint registries as a revision of the medial UKA, it is possible to perform it as though it were a primary procedure, with a small incision. Since the lateral compartment is being addressed as if a primary UKA, the procedure benefits from short tourniquet times and early hospital discharge. This patient is high risk for major surgery and opted for a smaller, safer procedure to avoid the risks associated with conversion to TKA.

It was discussed, during the consent process, that should the PFJ be worn or the ACL completely dysfunctional, the surgeon would have a low threshold for conversion to TKA. The previous UKA incision had been medial to the 

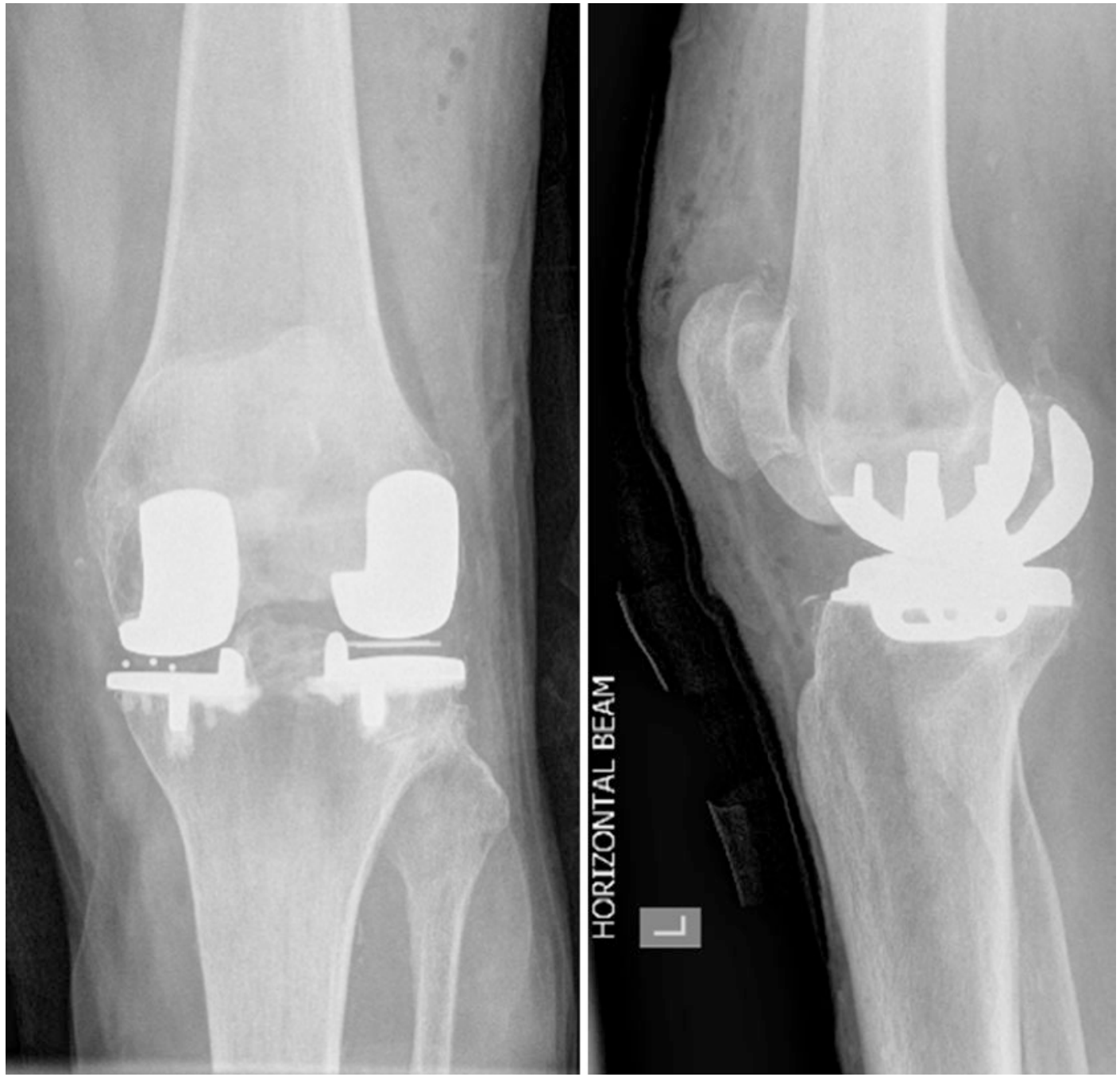

Fig. 21.8 Post-operative radiographs demonstrate conversion to Bi-UKA through the addition of a lateral UKA

midline, and therefore, a parallel lateral incision was made, leaving a $6-\mathrm{cm}$ skin bridge between the wounds. Had the previous incision been more midline, it would have been re-used, but a new lateral parapatellar arthrotomy made, to access the lateral compartment. The ACL was found to be degenerate but functional, which is not considered a contra-indication in elderly low-demand patients. The medial UKA was well fixed with minimal evidence of polyethylene wear, so it was left, though in high-functioning patients the polyethylene is often exchanged if signs of wear are evident. The tourniquet time was $48 \mathrm{~min}$, and the patient was discharged the following day. Post-operative radiographs (Fig. 21.8) demonstrate the Bi-UKA in situ. In this instance, a mobile bearing was used; however, due to the increased dislocation rate, a fixed bearing may be preferable in elderly, lowdemand patients.

Biazzo et al. compared 19 patients undergoing single-stage Bi-UKA to a matched cohort undergoing computer-assisted TKA, showing superior outcome in terms of function and stiffness on WOMAC indexes and equivalent KSS and WOMAC Arthritis Index (pain score) [20]. Single-staged Bi-UKA is associated with shorter hospital stays than TKA [32]. 


\subsection{Summary}

CPKA is more technically demanding than TKA in theory, but is associated with excellent postoperative outcomes and superior function [18]. It is suitable both for young, high-demand patients looking for excellent function and for higher risk patients, particularly in the revision setting, providing a safer, conservative alternative to TKA.

\section{References}

1. McAlindon TE, Snow S, Cooper C, Dieppe PA. Radiographic patterns of osteoarthritis of the knee joint in the community: the importance of the patellofemoral joint. Ann Rheum Dis. 1992;51(7): 844-9.

2. Ahlbäck S. Osteoarthrosis of the knee. A radiographic investigation. Acta Radiol Diagn (Stockh). 1968;(Suppl 277):7-72.

3. Ledingham J, Regan M, Jones A, Doherty M. Radiographic patterns and associations of osteoarthritis of the knee in patients referred to hospital. Ann Rheum Dis. 1993;52(7):520-6.

4. Duncan RC, Hay EM, Saklatvala J, Croft PR. Prevalence of radiographic osteoarthritis-it all depends on your point of view. Rheumatology (Oxford). 2006;45(6):757-60.

5. Johnson AJ, Howell SM, Costa CR, Mont MA. The ACL in the arthritic knee: how often is it present and can preoperative tests predict its presence? Clin Orthop Relat Res. 2013;471(1):181-8.

6. Duthon VB, Barea C, Abrassart S, Fasel JH, Fritschy D, Menetrey J. Anatomy of the anterior cruciate ligament. Knee Surg Sports Traumatol Arthrosc. 2006;14(3):204-13.

7. Bourne RB, Chesworth BM, Davis AM, Mahomed NN, Charron KD. Patient satisfaction after total knee arthroplasty: who is satisfied and who is not? Clin Orthop Relat Res. 2010;468(1):57-63.

8. Liddle AD, Judge A, Pandit H, Murray DW. Adverse outcomes after total and unicompartmental knee replacement in 101,330 matched patients: a study of data from the National Joint Registry for England and Wales. Lancet (London, England). 2014;384(9952):1437-45.

9. Cobb J. Osteoarthritis of the knee. Precise diagnosis and treatment. BMJ. 2009;339:b3747.

10. Garner A. van Arkel RJ, Cobb J. Classification of combined partial knee arthroplasty, Bone Joint $\mathrm{J}$. 2019;101-B(8):922-28.

11. Toliopoulos P, LeBlanc MA, Hutt J, Lavigne M, Desmeules F, Vendittoli PA. Anatomic versus mechanically aligned total knee arthroplasty for unicompartmental knee arthroplasty revision. Open Orthop J. 2016;10:357-63.
12. Thienpont E, Price A. Bicompartmental knee arthroplasty of the patellofemoral and medial compartments. Knee Surg Sports Traumatol Arthrosc. 2013;21(11):2523-31.

13. Palumbo BT, Henderson ER, Edwards PK, Burris RB, Gutierrez S, Raterman SJ. Initial experience of the journey-deuce bicompartmental knee prosthesis: a review of 36 cases. J Arthroplast. 2011;26(6 Suppl):40-5.

14. Wang H, Dugan E, Frame J, Rolston L. Gait analysis after bi-compartmental knee replacement. Clin Biomech (Bristol, Avon). 2009;24(9):751-4.

15. Argenson JN, Parratte S, Bertani A, Aubaniac JM, Lombardi AV Jr, Berend KR, et al. The new arthritic patient and arthroplasty treatment options. J Bone Joint Surg Am. 2009;91(Suppl 5):43-8.

16. Leffler J, Scheys L, Plante-Bordeneuve T, Callewaert B, Labey L, Bellemans J, et al. Joint kinematics following bi-compartmental knee replacement during daily life motor tasks. Gait Posture. 2012;36(3):454-60.

17. Parratte S, Ollivier M, Opsomer G, Lunebourg A, Argenson JN, Thienpont E. Is knee function better with contemporary modular bicompartmental arthroplasty compared to total knee arthroplasty? Short-term outcomes of a prospective matched study including 68 cases. Orthop Traumatol Surg Res. 2015;101(5):547-52.

18. Heyse TJ, Khefacha A, Cartier P. UKA in combination with PFR at average 12-year follow-up. Arch Orthop Trauma Surg. 2010;130(10):1227-30.

19. Cartier P, Sanouiller JL, Grelsamer R. Patellofemoral arthroplasty. 2-12-year follow-up study. J Arthroplast. 1990;5(1):49-55.

20. Biazzo A, Silvestrini F, Manzotti A, Confalonieri N. Bicompartmental (uni plus patellofemoral) versus total knee arthroplasty: a match-paired study. Musculoskelet Surg. 2019;103:63-8.

21. Tan SM, Dutton AQ, Bea KC, Kumar VP. Bicompartmental versus total knee arthroplasty for medial and patellofemoral osteoarthritis. J Orthop Surg (Hong Kong). 2013;21(3):281-4.

22. Romagnoli S, Marullo M, Massaro M, Rustemi E, D'Amario F, Corbella M. Bi-unicompartmental and combined uni plus patellofemoral replacement: indications and surgical technique. Joints. 2015;3(1):42-8.

23. Rolston L, Bresch J, Engh G, Franz A, Kreuzer S, Nadaud M, et al. Bicompartmental knee arthroplasty: a bone-sparing, ligament-sparing, and minimally invasive alternative for active patients. Orthopedics. 2007;30(8 Suppl):70-3.

24. Engh GA. A bi-compartmental solution: what the deuce? Orthopedics. 2007;30(9):770-1.

25. Stuyts B, Vandenberghe M, Bracht H, Fortems Y, Van den Eeden E, Cuypers L. Fracture of the tibial baseplate in bicompartmental knee arthroplasty. Case Rep Orthop. 2015;2015:1-5.

26. Steinert AF, Beckmann J, Holzapfel BM, Rudert M, Arnholdt J. Bicompartmental individualized knee replacement: use of patient-specific implants and instruments (iDuo). Oper Orthop Traumatol. 2017;29(1):51-8. 
27. Argenson JN, Chevrol-Benkeddache Y, Aubaniac JM. Modern unicompartmental knee arthroplasty with cement: a three to ten-year follow-up study. J Bone Joint Surg Am. 2002;84(12):2235-9.

28. Wunschel M, Lo J, Dilger T, Wulker N, Muller O. Influence of bi- and tri-compartmental knee arthroplasty on the kinematics of the knee joint. BMC Musculoskelet Disord. 2011;12:29.

29. Zanasi S. Innovations in total knee replacement: new trends in operative treatment and changes in peri-operative management. Eur Orthop Traumatol. 2011;2(1-2):21-31.

30. Parratte S, Pauly V, Aubaniac JM, Argenson JN. Survival of bicompartmental knee arthroplasty at 5 to 23 years. Clin Orthop Relat Res. 2010;468(1):64-72.

31. Lonner JH. Modular bicompartmental knee arthroplasty with robotic arm assistance. Am J Orthop (Belle Mead, NJ). 2009;38(2 Suppl):28-31.

32. Confalonieri N, Manzotti A, Cerveri P, De Momi E. Bi-unicompartmental versus total knee arthroplasty: a matched paired study with early clinical results. Arch Orthop Trauma Surg. 2009;129(9):1157-63.

33. Pradhan NR, Gambhir A, Porter ML. Survivorship analysis of 3234 primary knee arthroplasties implanted over a 26-year period: a study of eight different implant designs. Knee. 2006;13(1):7-11.

34. Shah SM, Dutton AQ, Liang S, Dasde S. Bicompartmental versus total knee arthroplasty for medio-patellofemoral osteoarthritis: a comparison of early clinical and functional outcomes. J Knee Surg. 2013;26(6):411-6.

35. Pritchett JW. Anterior cruciate-retaining total knee arthroplasty. J Arthroplast. 1996;11(2):194-7.
36. Andriacchi TP, Galante JO, Fermier RW. The influence of total knee-replacement design on walking and stair-climbing. J Bone Joint Surg Am. 1982;64(9):1328-35.

37. Neogi T, Felson D, Niu J, Nevitt M, Lewis CE, Aliabadi $\mathrm{P}$, et al. Association between radiographic features of knee osteoarthritis and pain: results from two cohort studies. BMJ. 2009;339:b2844.

38. Felson DT, Nevitt MC, Yang M, Clancy M, Niu J, Torner JC, et al. A new approach yields high rates of radiographic progression in knee osteoarthritis. J Rheumatol. 2008;35(10):2047-54.

39. Pandit H, Jenkins C, Gill HS, Barker K, Dodd CA, Murray DW. Minimally invasive Oxford phase 3 unicompartmental knee replacement: results of 1000 cases. J Bone Joint Surg. 2011;93(2):198-204.

40. Goodfellow J, O'Connor J, Pandit H, Dodd CA, Murray D. Unicompartmental arthroplasty with the Oxford knee. 2nd ed. Oxford: Goodfellow; 2016. p. 288 .

41. Price AJ, Svard U. A second decade lifetable survival analysis of the Oxford unicompartmental knee arthroplasty. Clin Orthop Relat Res. 2011;469(1):174-9.

42. Altuntas AO, Alsop H, Cobb JP. Early results of a domed tibia, mobile bearing lateral unicompartmental knee arthroplasty from an independent centre. Knee. 2013;20(6):466-70.

43. Pandit H, Mancuso F, Jenkins C, Jackson WFM, Price AJ, Dodd CAF, et al. Lateral unicompartmental knee replacement for the treatment of arthritis progression after medial unicompartmental replacement. Knee Surg Sports Traumatol Arthrosc. 2017;25(3):669-74.

Open Access This chapter is licensed under the terms of the Creative Commons Attribution 4.0 International License (http://creativecommons.org/licenses/by/4.0/), which permits use, sharing, adaptation, distribution and reproduction in any medium or format, as long as you give appropriate credit to the original author(s) and the source, provide a link to the Creative Commons license and indicate if changes were made.

The images or other third party material in this chapter are included in the chapter's Creative Commons license, unless indicated otherwise in a credit line to the material. If material is not included in the chapter's Creative Commons license and your intended use is not permitted by statutory regulation or exceeds the permitted use, you will need to obtain permission directly from the copyright holder. 